\title{
Rapid Defects Features Extract Techniques with Chirp Signal in Plate Structures
}

\author{
Fei Deng* \\ School of Electron and Information engineering \\ Ningbo University of Technology \\ Ningbo, China \\ *Corresponding author
}

\author{
Honglei Chen \\ School of Electrical and Electronic Engineering \\ Shanghai Institute of Technology \\ Shanghai, China
}

\begin{abstract}
This work presents a convenience means to get defects features with linear chirp signal and demonstrate it with finite element method and BP neural networks. Aluminum plate models with various kinds of defects modeling with numerical simulation software ABAQUS to get pattern recognition samples.In order to acquire tone burst responses at a wide range of frequencies, we take chirp signals as excitation. The defects scattering signals, which would be used to build sample library, are extracted from the calculated tone burst results. .Finally, two 2-layers BP neural networks are utilized to realize defects types and sizes identification. Test results show that this rapid defects features extract approach is useful to recognize the defect characteristics, although some misidentification exist.
\end{abstract}

Keywords-BP neural networks; chirp excitation; defects identification; plate style structures

\section{INTRODUCTION}

Plate style structures have been widely used in the area of civilian infrastructure, industrial and military aeronautics. Effectively detect and identify the defects are meaningful for nondestructive testing (NDT) and structures health monitoring (SHM). There are already many literatures about defects identification in recent years. HongTaoetal. [1]realized the delamination recognition in composite plates with BP neural networks which have various geometry sizes and positions, the defects features were characterized with inherent frequency. Gang etal. [2] successfully identified the size and position of cracks in isotropy platewith the amplitude information of Lame waves scattering signalin frequency domain by genetic algorithms. In[3], spectral element method and piezoelectric sensors array configuration were taken to do flaws recognition in aluminum plates. Piguangetal. [4] researched the defects indication in the frame structure with the natural frequency and BP network. Hindersetal.[5] extracted cracks features from the Lamb wave signals using wavelets and statistical pattern classification algorithms to identify flaw severity.

In this study, a more convenience approach is developed to do defects pattern identification analysis. Defects which have various shapes and physical sizes in aluminum plates are modeled with commercial software ABAQUS. The BP neural networks are chose as classifier, the sequences that consist by amplitudes of the first arriving wave packet, which calculated with Hilbert transform from different frequencies, are used as training and test samples. The cracks in testing samples have the similar geometry dimension or deflection degree with those in training samples. Finally, defects classification is realized successfully with the trained classifier.

\section{BASIC THEORY}

\section{A. Chirp Excitation}

Because the starting frequency, end frequency and the duration time of a linear chirp signal are apt to be controlled, there are already many studies about chirp signals used in NDT and SHM fields[6-9].Multiple narrowband and tone burst responses can easily be extracted from broadband chirp stimulate responses in experiments or simulations. Abroad band chirp excitation, which maybe be limited by Gaussian or Hanning window, could be effectively used to significantly improve the signal-to-noise ratio(SNR) without time domain averaging. The equation for such excitation is

$$
S_{c}(t)=w(t) \sin \left[f_{0} t+\frac{\pi B t^{2}}{T}\right]
$$

Where $f_{0}$ is the starting frequency, $T$ is the duration of chirp, and $B$ is the chirp bandwidth, $w(t)$ is a rectangle window spanning from 0 to $T$. Figure I shows the waveform of a linear chirp signal that will be used in the following finite element analysis. The frequency changes from $50 \mathrm{kHz}$ to $120 \mathrm{kHz}$ over a $58.7 \mu$ s window.

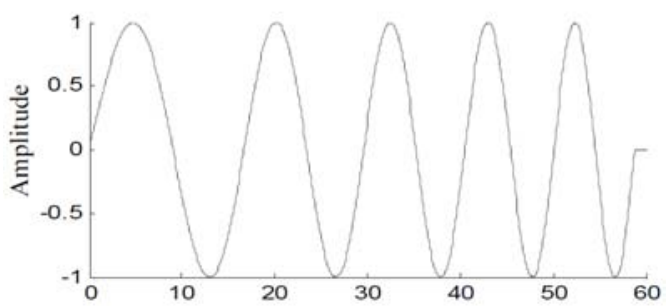

FIGURE I CHIRPSIGNAL FREQUENCY CHANGE FROM $50 \mathrm{kHzTO} 120 \mathrm{kHz}$ OVER A $58.7 \mu \mathrm{s}$ WINDOW

As described in [6], the response of a tone burst excitation in frequency domain can be calculated with the following equation:

$$
R_{d}(\omega)=R_{c}(\omega) \frac{S_{d}(\omega)}{S_{c}(\omega)}
$$

$\operatorname{Sd}(\omega)$ and $\operatorname{Sc}(\omega)$ are the tone burst and chirp signal in frequency domain, the responses of them are indicated with 
$\operatorname{Rd}(\omega)$ and $\operatorname{Rc}(\omega)$. If the excitation frequency falls in the bandwidth of the chirp, the simulate results of tone burst excitation in the time domain could be get easily by inverse Fourier transform operation of $\operatorname{Rd}(\omega)$.

\section{B. BP Neural Network}

$\mathrm{BP}$ (Back-Propagation) neural network is a kind of back propagate learning network that needs to be trained by the tutor Commonly, the network consists within put layer, hidden layer, and output layer. Patterns information are stored in the networks that indicated by the link weights and threshold between layers after repeating training with samples. Traditionally, every BP network has a pair of input layer and output layer, the number of sample points and classification code method decide the nodes number of them.

In this research, a 2-layers BP neural network is selected as classifier in this paper. The amplitude-frequency sequences that calculated from chirp excitation responses of models that have different kinds of cracks are used as input data of BP networks. The number of nodes, either in the middle layer or in the hidden layer, is over two times of the input layer. The number of the nodes in output layer is setting as the coding bitrate, which is according to the information need to be recognized, including the types and the sizes of defects. S style tangent function 'tansig' is set as the output function of hidden layer. The output function of the nodes in output layer is a $\mathrm{S}$ logarithmic function 'logsig', which would normalize the amplitude range as the closed unit interval $[0,1]$. The tangent function 'trainlm' is used to train the neural network, the target accuracy is setting as 0.002 and learning rate is 0.1 .

\section{NUMERICAL MODELS}

Several aluminum plates are modeled with 3D FE analysis (ABAQUS software). The geometry dimension of them is $600 \times 400 \times 2 \mathrm{~mm}^{3}$. Eight-noded $3 \mathrm{D}$ reduced integration solid brick elements C3D8R is used for model meshing. The dynamic problem is then solved with the explicit dynamic analysis. Figure II plotsLamb waves dispersion curves calculated analytically with the software DISPERSE. The phase velocity dispersion curves and the group velocity dispersion curves are plotted with black lines and gray lines respectively. As it shows, the velocity of $S_{0}$ mode is twice faster than $A_{0}$ mode. It means these modes can be easily separated when the Lamb waves propagates enough distance.

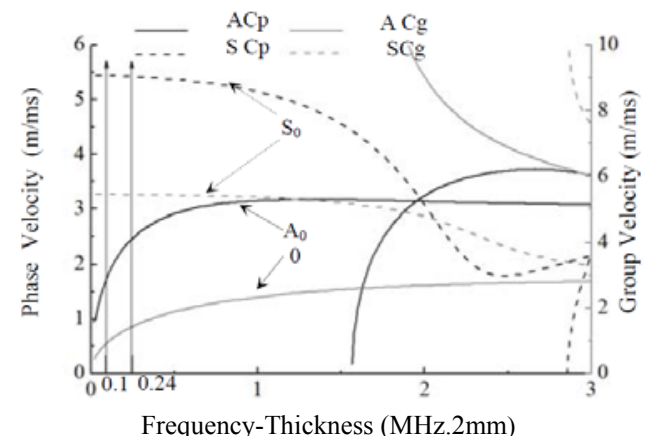

FIGURE II DISPERSION CURVES FOR A $2 \mathrm{mmTHICH}$ ALUMINUM PLATE
Figure III shows a model with a $7 \times 3 \mathrm{~mm}^{2}$ rectangular penetrant crack with Odegree offset to the $y$ axis. The original point is at the lower left corner of the plate, defect deflection center noted with character' $o$ ' is located at $(200,300)$. The chirp signal is loaded on the surface $\mathrm{F}$ with surface pressure. The $S_{0}$ Lamb wave is generated by applying out-of-plane nodal displacement to the surface point $R_{1}$ located at $(210,206)$. The elements size is $1.2 \times 0.5 \mathrm{~mm}^{2}$ to guarantee that the size of grids is less than $1 / 10$ of wavelength in any propagation direction. The total running time is $300 \mu \mathrm{s}$ and the step time is approximately $0.01 \mu \mathrm{s}$ in simulating.

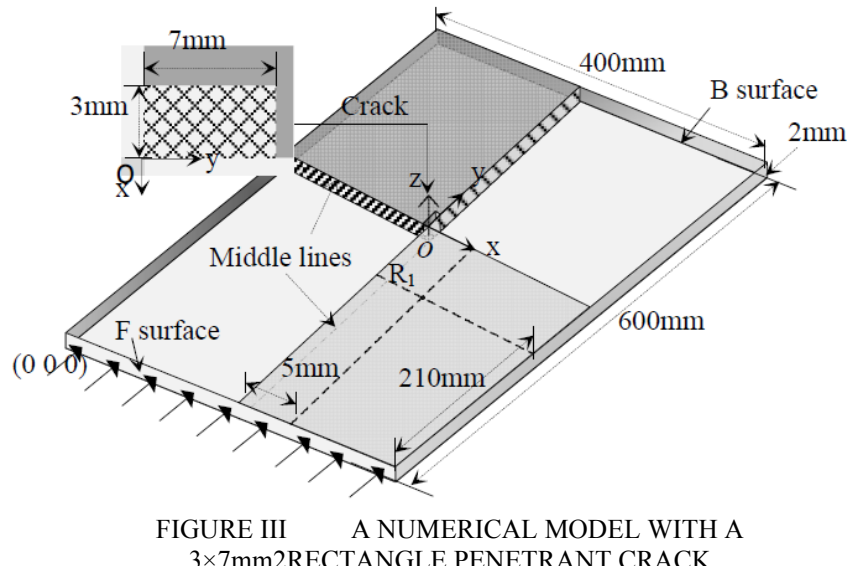

TABLE I DEFECTS INFORMATION IN TESTING SAMPLES

\begin{tabular}{cccccccc}
\hline $\begin{array}{c}\text { Lon } \\
\mathbf{g}\end{array}$ & $\begin{array}{c}\text { Width } \\
\text { /mm }\end{array}$ & Angle & Shape & $\begin{array}{c}\text { Lon } \\
\mathbf{g} \\
/ \mathbf{m m}\end{array}$ & $\begin{array}{c}\text { Width } \\
/ \mathbf{m m}\end{array}$ & $\begin{array}{c}\text { Angl } \\
\mathbf{e}\end{array}$ & Shape \\
\hline 7 & 3 & 10 & rectangle & 10 & 4 & 0 & ellipse \\
12 & 3 & 10 & rectangle & 11 & 3 & 85 & ellipse \\
6.5 & 3 & 45 & rectangle & 7 & 4 & 60 & ellipse \\
13 & 2 & 50 & rectangle & 13 & 2 & 50 & ellipse \\
7 & 4 & 60 & rectangle & 12 & 3 & 0 & ellipse \\
13.5 & 2.5 & 70 & rectangle & & & & \\
\hline
\end{tabular}

Three kinds of models, including the reference model, the training sample model and the testing sample model, are built to get the samples for pattern recognition analysis. The reference model has the same geometry dimension with the others just free of defect. In training models, the geometry dimensions of rectangular penetrant defects are $7 \times 3 \mathrm{~mm}^{2}$ and $13 \times 3 \mathrm{~mm}^{2}$, the long axis and short axis of ellipse defects are $7 \mathrm{~mm}$ and $13 \mathrm{~mm}$ respectively, and defect deflection angle is changing from Odegree to 90degree with 15degree intervals. Defects in testing models are close to those in training models in geometry dimension, some detailed information of the defects in testing models are shown in Table1.

\section{PATTERN RECOGNITION ANALYSIS}

\section{A. Features Extraction}

In order to extract features, there are four steps as following. At first, according to equation (2), calculate the tone burst simulated results in the specified frequency from chirp responses in different models with or without defects. As an example, the $110 \mathrm{kHz} 5$ cycles tone burst results are plotted in Figure IV. There are clear differences in the waveforms 
between $[84 \mu \mathrm{s} 185 \mu \mathrm{s}]$. The next step is to extract scattering signals by executing subtraction between detection signals on testing models (or training models) and baseline signals one by one. The typical scattering signal at $110 \mathrm{kHz}$ is shown in Figure $\mathrm{V}$ and the amplitude scale is at the level $1 e^{-15}$. We can find that three wave packets exist between the times scale $[84 \mu \mathrm{s}$ $185 \mu \mathrm{s}]$.After that, frequency-amplitude sequences are calculated from their Hilbert transforms at various frequencies. These sequences, as defects feature samples noted with character ' $A$ ', are consist with the maximum absolute amplitude in the envelopes of the scattering signals. Finally, do some pre-processon these sequences. Traditionally, samples always need to be converted into dimensionless data by normalized to eliminate units differences. In this paper, all data multiply $1 e^{14}$ to adjust their value levels.

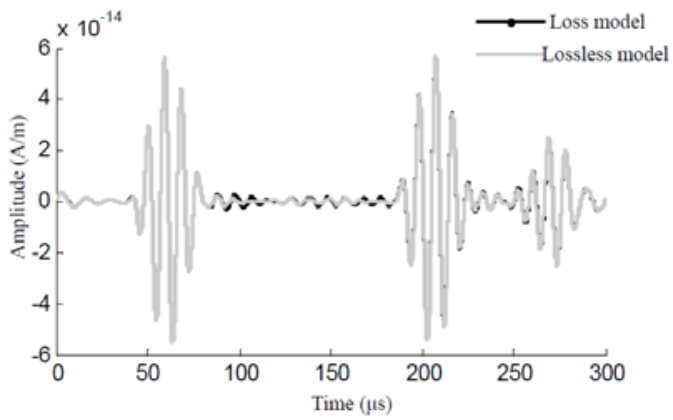

FIGURE IV COMPARISON OF $110 \mathrm{kHz}$ TONEBURSTRESPONSES CALCULATED FROM CHIRPRESPONSES

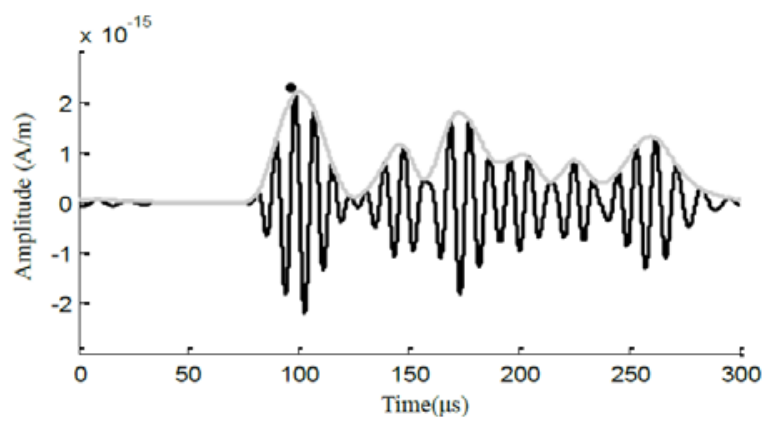

FIGURE V CALCULATED SCATTERING SIGNAL AT $110 \mathrm{kHz}$

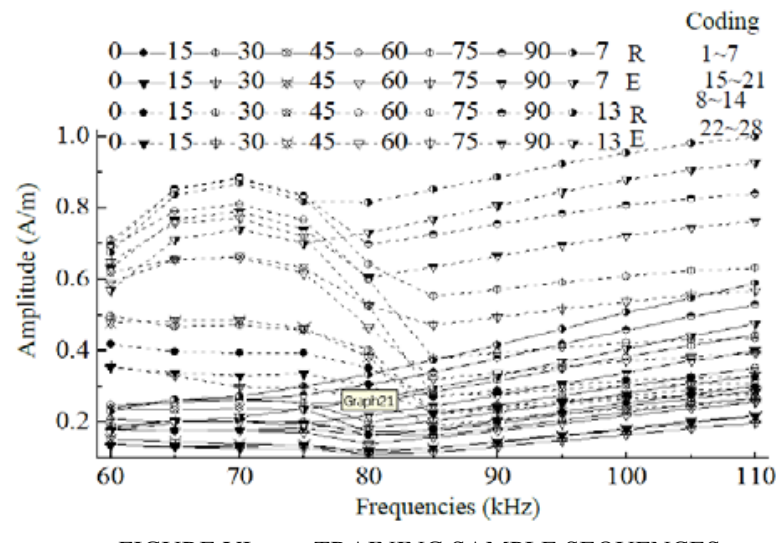

FIGURE VI TRAINING SAMPLE SEQUENCES
With the approach mentioned above, we get the training samples and testing samples in the frequency range spanning from $60 \mathrm{kHzto} 110 \mathrm{kHz}$ at $5 \mathrm{kHz}$ intervals. For any defects considered in this paper, the first reflection echo amplitudes at 11 selected frequencies are connected to a line, and 28 training sample lines are shown in Figure VI. In this figure, the rectangular (abbreviated as character ' $R$ ') and elliptic (abbreviated as character ' $E$ ') defects are denoted with solid line and dashed line respectively. It is evidence that the amplitudes and the frequency have nonlinear relationship, particularly when the frequencies are under $85 \mathrm{kHz}$. In addition to that, these curves showed a clear separation for the two types of typical defects examined in this paper. This separation character is conducive to the identification of defect types in the later steps.

According to the same method, the frequency-amplitude curves of different testing samples obtained too. Some curves are shown in Figure VII, in which the solid line represent the rectangular defect and the dashed line represent the elliptic defect. The main parameters, including the coding, deflection angle, length and width of the sample, are displayed atthe upper part of the Figure.

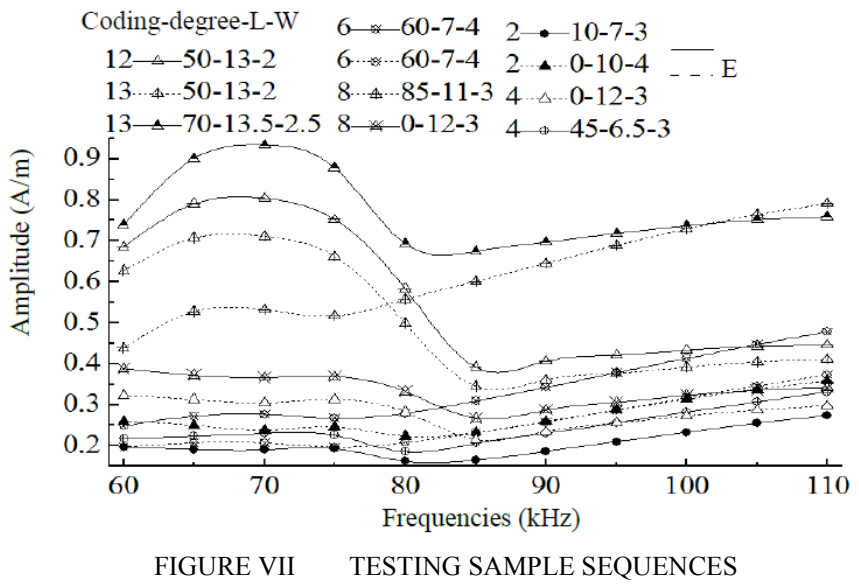

\section{B. Classifier Training and Testing}

1) Defects type identification: Because there are two kinds of defects, rectangle and ellipse, and each frequency-amplitude sequence has eleven elements in them, an BP neural network is designed with the structure of $11 \times 23 \times 2$ to do defect styles recognition. Parameters, such as output function and training precision etc. are set as described above. The output is a twodimensional element matrix, the rectangular and elliptic defects are denoted by $[1 ; 0]$ and $[0 ; 1]$ separately.

The training performance result, measured in terms of mean squared deviation in log scale, show that when the training has experienced 83 epochs, the output mean squared deviation is 0.00151026 which is smaller than the setting value 0.002 .Then the trained classifier is used for the testing samples. The network outputs will locate in the range of $\left[\begin{array}{ll}0 & 1\end{array}\right]$, so we use 'vec2ind' function to get the classification character which is corresponding to the location of the maximum value in the output vector and used to characterize defect types. The 
recognition results are described in the table2, and the first column lists the defects geometry information.

Testing results show that almost all the samples have been realized pattern recognition when the cracks only have $2 \mathrm{~mm}$ difference in geometry dimension between the training samples and the testing samples. However, the classifier gives wrong results at row 7 and row 8 . Take row 7 as an example, it should be an ellipse defect with $10 \times 4 \mathrm{~mm}^{2}$ Odegree deflection angle, but the classifier consider it as a rectangle. The inconsistencies attribute to the limited sample library. Only four defects designed with Odegree deflection angle exist in the sample library. Compared with these samples, there are at least $3 \mathrm{~mm}$ differences in the long axis length, which results in misidentifications.

TABLE II DEFECT TYPES RECOGNITION RESULTS

\begin{tabular}{cccc}
\hline $\begin{array}{c}\text { Defects } \\
\text { geometry }\end{array}$ & Shape & $\begin{array}{c}\text { Theoryres } \\
\text { ult }\end{array}$ & $\begin{array}{c}\text { Identification } \\
\text { result }\end{array}$ \\
\hline $10-7-3$ & rectangle & 1 & 1 \\
$0-12-3$ & rectangle & 1 & 1 \\
$60-7-4$ & rectangle & 1 & 1 \\
$70-13.5-2.5$ & rectangle & 1 & 1 \\
$45-6.5-3$ & rectangle & 1 & 1 \\
$50-13-2$ & rectangle & 1 & 1 \\
$0-10-4$ & ellipse & 2 & 1 \\
$85-11-3$ & ellipse & 2 & 1 \\
$60-7-4$ & ellipse & 2 & 2 \\
$50-13-2$ & ellipse & 2 & 2 \\
$0-12-3$ & ellipse & 2 & 2 \\
\hline
\end{tabular}

2) Defects dimension identification: The same sample library is used for the rectangle penetrant defects dimension identification analysis. There are 14 training samples and 6 testing samples.AnBP neural network with the structure of $11 \times 23 \times 14$ is built to appraise this approach. The target vector' $\mathrm{T}$ is a $14 \times 14$ element matrix, the row number of element one in the vector is indicating the defect type. For example, when the element one at the seventh row, it refers to the rectangle defects with $7 \times 3 \mathrm{~mm} 2$ and 90 deflection degree, as shown in Figure VI.

The classifier is repeating trained with the training samples until it reaches the setting precision. After the test with the testing samples, the identification results are shown in Table3. The first column lists the defect information of testing samples. The defects information of most closely samples which are found from the training sample library by the classifier are listed in the fourth column. The theory result column shows the defects coding as drawn in Figure VI, there are perfect consistencies with identification result column. When the defect of testing sample has similar geometry information with some samples in training library, this approach shows its effectiveness and robustness.
TABLE III DEFECTS DIMENSION RECOGNITION RESULTS

\begin{tabular}{cccc}
\hline defects info & $\begin{array}{c}\text { identification } \\
\text { result }\end{array}$ & theory result & defects \\
\hline $10-7-3$ & 2 & 2 & $15-7-3$ \\
$0-12-3$ & 8 & 8 & $0-13-3$ \\
$60-7-4$ & 5 & 5 & $60-7-3$ \\
$70-13.5-2.5$ & 13 & 13 & $75-13-3$ \\
$45-6.5-3$ & 4 & 4 & $45-7-3$ \\
$50-13-2$ & 11 & 11 & $45-13-3$ \\
\hline
\end{tabular}

\section{CONCLUSION}

This paper present a simple means to extract defects features with the linear chirp excitation and proven by numerical simulations. Two kinds of defects, rectangle and ellipse, are used to verify this approach with BP neural networks. From this study, the following conclusions can be drawn:

1) Take the linear chirp signal as the excitation is an efficient and convenient method to get abundant information used for defect identification;

2) This method could identify the defect types, geometry dimensions and deflecting angle of defects if there has appropriate samples used to train the classifier, BP networks;

3) As the testing results shows, when the physical dimension difference between the testing effect and the training samples is over $2 \mathrm{~mm}$, misidentification maybe happen. So, it is necessary to build a comprehensive training sample library.

\section{ACKNOWLEDGMENT}

Gratefully acknowledge and appreciate the support of the project of National Natural Science Fund, No.11202137, Shanghai City Board of Education Science and Technology Innovation Fund Project, No.13YZ119, and Ningbo University of Technology Research Fund Project, No. 0080011540161.

\section{REFERENCES}

[1] W. Hongtao, L. Lifeng, Z. Laishui. Investigation of Delamination Detection for CompositeStructures Based on Modal Analysis and BP Neural Network[J].China mechanical engineering, 2005, 16(3): 239-242.

[2] Y. Gang , Z. Li. Damage identification for plate-like structure using genetic algorithmsand scattered Lamb waves[J].Journal of Vibration Engineering, 2008, 20(3): 291-296.

[3] P. Haikuo, M. Guang, L. Fucai. A piezoelectric transducer arraybasedmethod for damage detection in plate structures[J].Journal of vibration and shock.2009, 28(9): 56-59.

[4] W. Piguang, G. Zhimou ,Z. Juan. Damage detection on concrete of single-story frame based on BP Neural Networks[J].Journal of institute of disaster prevention.2011, 13(2): 32-37.

[5] M. K.Hinders, C. A. Miller. Intelligent feature selection techniques for pattern classification of Lamb wave signals[C]. 40th Annual Reviewof Progressin Quantitative Nondestructive Evaluation: Incorporating the 10th International Conference on Barkhausen Noise and Micromagnetic Testing. AIP Publishing, 2014, 1581(1): 294-301.

[6] J. E.Michaels, S. J.Lee, J. S.Hall. Multi-mode and multi-frequency guided wave imaging via chirp excitations[C]. SPIE Smart Structures and Materials Nondestructive Evaluation and Health Monitoring. International Society for Optics and Photonics, 2011: 79840I-79840I-11.

[7] C. Honglei, D. Fei, Z. Xi. Defect Imaging via Chirp Signal Excitation in Plate[J]. Advanced Materials Research, 2014, 846: 826-830.

[8] A.J. Croxford, O. Putkis, P.D. Wilcox, Efficient guided wave SHM baselinecapture and selection, in: F.-K. Chang (Ed.), Proceedings of the 
8thInternational Workshop on Structural Health Monitoring, DEStechPublications, Inc., Lancaster, PA, 2011, pp. 2067-2074.

[9] Z. Yang, H. Cunfu, W. Bin. Chirp signal and its application in ultrasonic guided wave inspection[J]. Chinese Journal of Scientific Instrument,2013,3(34):552 558

[10] S. Zhongqing and Y. Lin. Identification ofDamage UsingLamb Waves from fundamentals to applications. Lecture notes in applied and computational mechanics,2009,Vol. 48, 1st Edition, London, Springer.

[11] D. Fei,C. Honglei,Z. Xi. Pipe Structure Detection Based on Broadband Guided Wave excitation[J]. Journalof Mechanical Engineering. 2014,50(18):23-28 